\title{
La poésie sur-le-champ (de bataille). Waterloo vu de part et d'autre de la Manche
}

Joseph-Désiré Court (1797-1865), futur conservateur du Musée des Beaux-Arts de sa ville natale, Rouen, a tôt fait preuve d'une vocation et de talents artistiques. Parmi les peintures de jeunesse qu'il réalisa bien avant son prix de Rome, une mention spéciale doit être décernée à La Lettre de Waterloo $^{1}$ (1816). Les yeux clos, pâle comme la mort, une femme éplorée serre contre elle son enfant. On comprend que l'époux et père a rendu l'âme sur le champ-de-bataille, sans qu'il soit nécessaire de montrer la moindre arme, le moindre emblème guerrier, si ce n'est la légion d'honneur, à gauche, décernée à titre posthume. Le programme iconographique, en ces années de grandes pertes militaires, est traité plus tard par d'autres, comme Claude-Marie Dubufe avec La Lettre de Wagram $\left(1828^{2}\right)$. Il n'est pas nécessaire d'être pour ou contre l'Empire pour comprendre l'émotion qui se dégage de ces scènes.

Les années postrévolutionnaires ont vu une floraison d'élégies sur les morts de proches dont l'inspiration sentimentale pourrait être rapprochée de ces deux tableaux. En matière poétique, on aurait pu s'attendre, en tout premier lieu, à une poésie de déploration des morts et de la défaite, une poésie du souvenir et du deuil, un équivalent textuel de l'esthétique de l'émotion qui se dégage de l'œuvre de Court. Waterloo, pourtant, du moins d'après les textes que j'ai pu consulter, n'a pas suscité un élan poétique comparable. Si j'ai voulu commencer par ce manque, c'est qu'il me semble que les réactions versifiées à la bataille «à chaud ${ }^{3}$ » en quelque sorte donnent des clefs pour une lecture historique de l'événement.

\section{Un nom mal né pour la poésie}

La rareté des poèmes écrits au lendemain de la bataille par les Français - j'écarte les vers apocryphes de Napoléon lui-même ${ }^{4}-$ me semble pouvoir s'expliquer de différentes manières. Il y a tout d'abord en français un problème qu'il n'y a pas en anglais, celui du mot lui-même. Les sonorités du toponyme ne sont pas naturelles pour un francophone, en témoignent le problème $\mathrm{du}$ 《 $\mathrm{W}$ » initial, mais aussi la question des voyelles finales. Que faire de ces deux «o »? En juin 1815, un royaliste adolescent célèbre la fin de l'Empire et fait donc de Waterloo une victoire pour une certaine France.

Champs de Waterloo, bataille mémorable,

Jour à la fois pour nous heureux et déplorable,

Qui pourrait exprimer votre sanglante $h .$.

Et du Corse trembl....

Frém... ${ }^{5}$

Il est des noms, comme l'écrit Roucher dans ses Mois en 1779, «mal né[s] pour la poésie ». «Wat-ter-lo-o », avec ses quatre syllabes, pouvait sembler être de ceux-là. On ne s'étonne pas, au vu de la jeunesse du poète - il avait treize ans - et du manque de maniabilité du terme, que le morceau fragmentaire cité ci-dessus n'ait pas vu le jour. Esquissé en alexandrins et

\footnotetext{
${ }^{1}$ Rouen, Musée des Beaux-Arts, Inv. : 2007.10.1.

${ }^{2}$ Rouen, Musée des Beaux-Arts, Inv. : 2004.4.1.

${ }^{3}$ Je traiterai avant tout de poèmes au moins en partie composés dans l'année qui a suivi la bataille.

${ }^{4}$ « Le nombre mais surtout la trahison funeste,/ Des héros de la France a dévoré le reste./ L'Europe en a frémi d'un long étonnement.../ Le fier Anglais, surpris des honneurs qu'on lui rend,/ Se demande en tremblant s'il a vaincu la France, /Si de Napoléon il brise la puissance,/ Si dans Waterloo nos soldats immortels/ Ont perdu dans un jour leurs lauriers éternels. » Voir L'Intermédiaire des chercheurs et des curieux du 20 mars 1894 selon lequel le poème en facsimilé aurait été exposé chez Painparé au Palais-Royal le 20 août 1821 et mis en vente au prix de 30 francs. ${ }^{5}$ Voir Pierre Laforgue, «Waterloo en poésie ou Casimir Delavigne, Cambronne et Hugo », Cahiers RoucherAndré Chénier 19 (2000), p. 197-205.
} 
vraisemblablement mutilé tardivement selon Jean-Marc Hovasse ${ }^{6}$, il est l'œuvre de Victor Hugo. On peut souligner qu'on trouve chez le Belge Joseph Le Mayeur, dans le texte (mais pas le titre) de son Ode sur la bataille de Waterloo ou de Mont Saint-Jean (Bruxelles, 1816), qui se réjouit vivement de la fin de l'Empire et assure que "Godefroy [de Bouillon] vit dans Wellington», la graphie «Waterlo» et on aura plus tard chez Nerval l'orthographe «Waterlô », nouveaux témoignages de la difficulté de faire entrer en poésie ce toponyme. On peut aussi citer les Couplets sur la journée de Waterloo de Béranger ${ }^{7}$ à propos du « dernier jour de gloire et de revers » dont le poète écrit, en guise de refrain, "Son nom jamais n'attristera mes vers. » Voici l'avant-dernière strophe :

Périsse enfin le géant des batailles !

Disaient les rois; peuples, accourez tous.

La Liberté sonne ses funérailles ;

Par vous sauvés, nous régnerons par vous.

Le géant tombe, et ces nains sans mémoire

À l'esclavage ont voué l'univers.

Des deux côtés ce jour trompa la Gloire,

Son nom jamais n'attristera mes vers.

Rejeté du poème, comme refusé par la mémoire du poète, Waterloo n'a pas droit de cité dans ses vers ${ }^{8}$. Une brochure de 16 pages de Saintine contourne, elle, la difficulté, en s'intitulant Hommage aux braves morts le 18 juin 1815, au mont Saint-Jean; suivi de Le Suicide, pièce élégiaque; de l'Aigle et les Lis etc ${ }^{9}$.

\section{Bataille du nom et nom de la bataille}

Il n'y a pas forcément qu'une simple résistance poétique à l'œuvre. Si Grégoire disait avoir créé le mot «vandalisme » pour tuer la chose, le nom même de Waterloo est porteur de tensions. Il n'arrange pas la poésie française, mais il aurait été promu par Wellington dans le cadre d'une opération de communication qui lui donnait le beau rôle, et contre le nom français de La Belle Alliance ${ }^{10}$ soutenu par Blücher pour des raisons symboliques ${ }^{11}$. C'est du moins ce qu'indiquent des lettres du poète anglais Robert Southey. Quelques semaines après les combats, il écrit :

The name which Blucher has given it [the battle] will do excellently in verse, the field - of Fair Alliance, but I do not like it in prose, - for we gave them such an English threshing that the name of the battle ought to be one which comes easily out of an English mouth. - If you can help me to any ${ }_{x x x x x x x x}$ I shall know how to use it ${ }^{12}$.

\footnotetext{
${ }^{6}$ Communication orale à l'occasion du colloque publié ici. Selon Jean-Marc Hovasse, le manuscrit a été mutilé probablement par Hugo, revenant sur un poème dont les sentiments politiques ne lui convenaient plus.

${ }^{7}$ Incipit du poème : «De vieux soldats m’ont dit : Grâce à ta Muse ».

${ }^{8}$ En opposition directe avec cela, Quinet écrira plus tard « Moi, je suis Waterloo ! » dans la série des batailles évoquées au sein du poème «Les Veuves».

${ }^{9}$ Je n'ai pas eu accès à l'ouvrage. Voici le compte rendu qu'en donne l'Almanach des Muses en 1816 : «Sans doute l'on n'est point coupable en défendant son pays : on serait criminel, au contraire, en ne le défendant pas. Mais ces braves morts au Mont-Saint-Jean, quelle cause avaient-ils abandonnée, et quelle cause ont-ils suivie ? Déplorons leur aveuglement, pleurons leur mort, et sachons gré à M. de Saintine de l'hommage qu'il rend, en bons vers, à leur mémoire. »

${ }^{10}$ Un poème, que je n'ai pas pu consulter, s'intitule ainsi : La Belle Alliance, ode dédiée à S.A.R. la Princesse d'Orange de Couquebeau alias Bouqueau (1816).

11 «Belle Alliance » est aussi le nom du consortium qui est à l'origine du Mémorial 1815.

${ }^{12}$ Lettre 2633. MS : Huntington Library, RS 254. Texte publé dans Charles Cuthbert Southey (éd.), Life and

Correspondence of Robert Southey, 6 vols (London, 1849-1850), IV, pp. 119-120 [in part; dated 10 July 1815].
} 
Wellington avait inscrit Waterloo en-tête de sa dépêche sur le combat car c'est de là qu'il l'écrivait. La presse anglaise entérine aussitôt le recours à ce nom-là pour désigner la bataille, le Times du 23 juin 1815 se réjouit ainsi de ce qui est désigné comme la « Glorious victory of Waterloo ».

En décembre de la même année, Southey assure que le nom de Waterloo n'était pas celui qu'on aurait pu s'attendre à retenir ${ }^{13}$ et explique son propre point de vue de poète engagé dans une célébration versifiée :

I have written 200 lines of my poem, - it grows under my hands \& will very probably extend to 1000. The notes will extend it to a little volume, \& it is likely to do me credit. I call it La Belle Alliance because (between ourselves) the name of Waterloo was given to the battle by the Duke of Wellington in a spirit of the lowest \& vilest jealousy, - of which when we meet you shall have damning proofs. Waterloo is three miles from the nearest point of action, $\&$ the battle might as well have been called the battle of $\mathrm{S}^{\mathrm{t}}$ Helens Auckland ${ }^{14}$.

Dans une strophe de son poème Southey évoque le nom anglais de la bataille, laissant ainsi entendre qu'une autre appellation aurait pu s'imposer :

Here, where the woods receding from the road

Have left on either hand an open space

For fields and gardens, and for man's abode,

Stands Waterloo; a little lowly place,

Obscure till now, when it hath risen to fame,

And given the victory its English name.

\section{Des héros en nombre}

Rien donc, ne semble aller dans le sens d'une poésie de Waterloo en français. Malgré tout, s'il y a un poème français important écrit au lendemain de la bataille, c'est celui d'un jeune ténor des concours académiques de ces temps-là, Casimir Delavigne (1793-1843), dont l'Ode à la vaccine a obtenu un accessit décerné par l'Institut en 1815. Sa première Messénienne, qui consacre sa réputation de poète, et qui, composée en juillet $1815^{15}$, fut couronnée d'un succès immense, porte justement sur «La Bataille de Waterloo ». Ses vers plaident pour une forme d'unité nationale dans un deuil collectif vu comme l'acte fondateur d'une nouvelle gloire française. Le poète n'esquive pas pour autant les aspects proprement militaires et il montre par une espèce de prétérition la souffrance de ceux qui se sont battus pour la patrie de tous les Français, qu'ils soient royalistes, napoléonistes ou républicains.

Cachez-moi ces soldats sous le nombre accablés,

Domptés par la fatigue, écrasés par la foudre,

Ces membres palpitants dispersés sur la poudre,

Ces cadavres amoncelés !

Éloignez de mes yeux ce monument funeste

\footnotetext{
Nous avons consulté l'édition en ligne des lettres de Southey à laquelle nous renvoyons pour toutes nos citations de cette correspondance : http://www.rc.umd.edu/editions/southey_letters/Part_Four.

${ }^{13}$ Le musée consacré à la bataille a été baptisé « Mémorial 1815 » comme pour esquiver la question chargée du nom à donner au lieu de mémoire.

${ }^{14}$ 2684. Robert Southey to Thomas Southey, 17 December 1815. - St Helen Auckland est un village au Nord-Est de l'Angleterre.

${ }^{15}$ Voir Victoires, Conquêtes des Français de 1792 à 1815 - Couronne poétique, Paris, Panckoucke, 1821, p. 320 .
} 
De la fureur des nations ;

Ô mort ! Epargne ce qui reste !

Aigles et étendards sont souillés d'une fange sanglante. Dans le chaos qu'est la plaine de Waterloo il n'y a plus que « Du sang, des débris et des morts. » Le poème de Delavigne réussit à rendre la défaite honorable. Il rappelle les exploits des guerriers français que l'ennemi même paraît respecter :

Les voilà ces héros si longtemps invincibles !

Ils menacent encor les vainqueurs étonnés !

Glacés par le trépas, que leurs yeux sont terribles!

Que de hauts faits écrits sur leurs fronts sillonnés!

Ils ont bravé les feux du soleil d'Italie,

De la Castille ils ont franchi les monts ;

Et le nord les a vus marcher sur les glaçons

Dont l'éternel rempart protège la Russie.

Ils avaient tout dompté...

Le drame serait pour ces soldats valeureux, d'être morts au champ d'honneur certes, mais à l'occasion d'une défaite, eux qui méritaient la victoire. Toute la fin du poème est une déploration des divisions internes de la France qui rendent chacun suspect à son voisin et fragilisent le pays entier face au reste du monde :

Le glaive étincelant dans nos mains infidèles,

Ensanglante le sein qu'il devrait protéger.

L'ennemi cependant renverse les murailles

De nos forts et de nos cités.

Delavigne en appelle à l'unité nationale. Il réunit dans un même souffle Bovines ou Bouvines, une victoire de Philippe-Auguste, et Austerlitz qu'il fait rimer avec « lis ». Il propose ainsi par le jeu de l'écho sonore une version performative de l'union qu'il prône :

Il est temps d'immoler au bonheur de la France

Cet orgueil ombrageux de nos opinions.

Etouffons le flambeau des guerres intestines.

Soldats, le ciel prononce, il relève les lis ;

Adoptez les couleurs du héros de Bovines,

En donnant une larme aux drapeaux d'Austerlitz.

France, réveille-toi !

L'appel au rassemblement se trouve également dans un important texte contemporain de Delavigne, La Découverte de la vaccine, qui aboutissait à une célébration d'une France unie par les beaux-arts plutôt que la puissance militaire. La force du poème de Delavigne, qui a un souffle épique certain, est de confronter la réalité sordide de la bataille en la passant par le filtre du style considéré comme noble, mais d'aboutir à une célébration ni des vaincus, ni des vainqueurs, mais bien de toute une France, exsangue, appelée à se relever ensemble. S'il n'y a pas d'épisode personnel analogue à l'esthétique des tableaux dont j'ai parlés, c'est aussi que Delavigne inscrit sa réflexion sur un autre plan. Il a également la particularité d'évacuer de son poème toute mention de Napoléon, lequel reste un héros de chansons populaires et d'images d'Épinal ${ }^{16}$. Il introduit un contrepoint, une sorte de valeur partagée des combattants par le biais

\footnotetext{
${ }^{16}$ Voir par exemple « PLEUREZ LES DEUX NAPOLEON. / OU / L'HISTOIRE DU ROI DE ROME. / Air connu. / Par ses succès, par sa vaillance (. . .) Pleurez les deux Napoléon. (m. g.) ; La bataille de Waterloo (...)
} 
d'un élément documentaire récurrent, la réplique prêtée à Cambronne, qui en rejeta la paternité ${ }^{17}$.

Le bataillon sacré, seul devant une armée,

S'arrête pour mourir.

C'est en vain que, surpris d'une vertu si rare,

Les vainqueurs dans leurs mains retiennent le trépas.

Fier de le conquérir, il court, il s'en empare ;

La garde, avait-il dit, meurt et ne se rend pas.

Cette phrase honorable rappelée par Casimir Delavigne est le point focal de bien d'autres célébrations versifiées du combat. En 1821, des vers d'un certain M. Lefèvre, de Rouen, dont je ne connais pas la date de composition, mais qui ont vraisemblablement été écrits peu après la bataille, paraissent - tout comme ceux de Delavigne que je viens de citer ou encore ceux de Béranger sur la fin des combats -, dans Victoires, Conquêtes des Français de 1792 à 1815 Couronne poétique ${ }^{18}$. Intitulés, «Dernier cri de la garde impériale », les trois huitains du Normand terminent sur le même vers, «La garde meurt, elle ne se rend pas ${ }^{19}$ ». Je vous livre la dernière strophe :

Dix rois ligués ont fait fléchir ta tête,

Français, trop fier de les avoir vaincus ;

Pour t'affranchir du joug de leur conquête,

Tu tenterais des efforts superflus ;

Mais si jamais l'heure de la vengeance

Vient à sonner, magnanimes soldats,

Ralliez-vous à ce cri de vaillance :

«La Garde meurt, elle ne se rend pas».

Une dizaine d'années après la bataille, Nerval reprendra dans «Waterloo » (1826) l'une des expressions associées à ce combat, mais qui est aussi porteuse d'un écho du Chant du départ de Marie-Joseph Chénier :

Tous, d'un commun accord, ont juré noblement

De vaincre ou de mourir pour la cause commune.

mais c'est par notre valeur. (b. m.) ; LE REVE DE MARIE-LOUISE / OU L'ENFANT DU MALHEUR. / Air de maman dort. /À l'ombre d'un antique chêne (. . .) ou est tu l'enfant du malheur. (sic) (h. d.) ; LES ADIEUX DU FILS DE / NAPOLEON. / Air de liberté sainte. / Ô mort déjà quoi ta faulx nous (. . .) Français vengeons, ces deux illustres morts. (b. d.) ; À PARIS. -Chez JULIENNE Fabricant d'Images, rue Saint-Merry. N. 37 (b. m.) ; Imp. de Poussin (b. d.) »

${ }^{17}$ Michel Balisson de Rougemont paraît être le premier à avoir cité cette réplique le 24 juin 1815 dans un article du Journal général de la France.

${ }^{18}$ Tome dernier, p. 319.

${ }^{19}$ Dans la mesure où il n'est guère connu, nous reproduisons ci-dessous le poème entier :

« Ils ne sont plus, les fils de la victoire !/ Mars a trahi leurs efforts et nos voux !/ Pleurez, Français, l'appui de votre gloire/ Est descendu dans la tombe avec eux ;/ À leur valeur l'Anglais rendant hommage,/ Voulut en vain les soustraire au trépas,/ Les preux ont dit, en volant au carnage :/ 'La Garde meurt, elle ne se rend pas'.// Toi qui deux fois leur dus le diadème,/ Toi qui, sans eux, eus gémi dans les fers,/ Napoléon, à cette heure suprême,/ Te verra-t-on partager leurs revers ! .../ Ils sont tombés les héros de la France,/ Et toi tu fuis !... au milieu des combats ;/ Tu fus donc sourd à ce cri de vaillance :/ 'La Garde meurt, elle ne se rend pas'.// Dix rois ligués ont fait fléchir ta tête,/ Français, trop fier de les avoir vaincus ;/ Pour t'affranchir du joug de leur conquête,/ Tu tenterais des efforts superflus ;/ Mais si jamais l'heure de la vengeance/ Vient à sonner, magnanimes soldats,/ Ralliez-vous à ce cri de vaillance :/ 'La Garde meurt, elle ne se rend pas'. » 
Nerval écrit encore : «La garde, ont-ils crié, meurt et ne se rend pas !» Implicitement, Cambronne, et non Napoléon, devient le héros - Lefèvre d'ailleurs laisse entendre qu'il eût été plus glorieux pour l'empereur de rendre l'âme sur le champ de bataille. Dans ce ralliement autour d'un cri honorable qui aurait pu être celui d'un républicain, comme d'un monarchiste, entre engagement pour une cause et respect d'un devoir, il y a une tentative pour certains de défendre un idéal politique précis, pour d'autres, comme Delavigne, de soutenir une unité nationale. Il y a ainsi, implicitement, une tendance qui ramène vers un centre qu'on essaye de consolider alors que les textes anglais dont je vais parler sont, à mon sens, centrifuges.

\section{Des vers de terrain}

Dès la nouvelle de la victoire des alliés et de la déroute de Napoléon, les muses britanniques sont en émoi. Le poète Robert Southey écrit ainsi à un ami, John Rickman, vers le 5 juillet $1815^{20}$. Il est avide de nouvelles. La bataille est la plus épique des temps modernes à s'être jouée ailleurs que sur les flots. L'éditeur Murray, flairant l'affaire commerciale, souhaite publier des textes là-dessus très vite. Southey s'y met d'emblée. Il se rendra en octobre sur les lieux, après avoir assisté, en Angleterre, à des célébrations mémorables.

Si le pèlerinage sur le champ de bataille s'est développé, c'est sans doute pour plusieurs raisons dont l'une est pragmatique et simple : pendant la Révolution, puis durant les guerres napoléoniennes, il avait été déconseillé aux Britanniques de voyager sur le continent lors d'excursions touristiques. Le champ de bataille où eut lieu la victoire décisive contre l'ogre corse devait tout naturellement s'inscrire dans un grand tour en version moderne. En plus des antiquités romaines, il faudrait désormais voir l'endroit où venait de se décider le sort de l'Europe. C'est vrai pour un jeune homme comme Walter Scott, qui était écossais. Il n'avait jamais mis pied hors des îles britanniques. Dès l'été 1815, il se dirigeait vers ce qui n'était pas encore la Belgique telle que nous la connaissons. Il savait que l'intérêt de ses compatriotes serait vif pour de véritables reportages sur les lieux des combats. Il composa un volume en prose intitulé Paul's Letters to his Kinsfolk (1816) à partir d'entretiens avec des témoins et de ses propres impressions. Il est l'un des premiers Britanniques à s'être rendu sur les lieux en tant que touriste. Il a eu pour cicérone des militaires qui avaient été présents et pouvaient le renseigner sur les événements tels qu'ils s'étaient passés. Il évite, grâce à cette approche, d'avoir à condamner sans appel l'empereur vaincu. Au contraire, il reconnaît ses qualités de stratège entre autres ${ }^{21}$ - il ne traite pas non plus les Français comme des ennemis lors de la suite de son reportage qui le voit traverser l'hexagone. Il a rencontré Wellington, comme il le raconte, et il donne au général anglais un rôle prépondérant, faisant de lui le vainqueur de Waterloo ce qui reste la manière dont la plupart des Britanniques considèrent encore aujourd'hui la bataille - en minorant l'implication de Blücher. Lors de son évocation du site, Scott documente aussi les débuts du tourisme à Waterloo alors que les traces des combats sont encore visibles. Il mentionne l'exploitation des victimes et la vente des reliques. Paul's Letters to his Kinsfolk fut très favorablement accueilli par un public avide d'en savoir plus sur le continent et sur la bataille qui avait mis fin à l'Empire.

Si le volume en prose est documentaire, Scott a des ambitions littéraires plus élevées et compose un long poème, The Field of Waterloo (traduit en français en 1821 par Defauconpret). Il y rend compte de son expérience de recherche d'indices dans le paysage même au moment où il découvre le champ de bataille :

\footnotetext{
${ }^{20}$ Lettre 2633. MS: Huntington Library, RS 254 reproduite dans Charles Cuthbert Southey (éd.), Life and Correspondence of Robert Southey, 6 vol. (Londres, 1849-1850), IV, p. 119-120 [en partie datée du 10 juillet $1815]$.

${ }^{21}$ En 1827, Scott devait publier une vie de Bonaparte qui, là encore, est loin d'être fondée sur des jugements à l'emporte-pièce.
} 
Now, see'st thou aught in this lone scene

Can tell of that which late hath been? -

A stranger might reply,

"The bare extent of stubble-plain

Seems lately lightened of its grain;

And yonder sable tracks remain

Marks of the peasant's ponderous wain,

When harvest-home was nigh.

On these broad spots of trampled ground,

Perchance the rustics danced such round

As Teniers loved to draw;

And where the earth seems scorched by flame,

To dress the homely feast they came,

And toiled the kerchiefed village dame

Around her fire of straw."

La réponse vient très vite. Ce qui a été moissonné l'a été non par une faux, mais par des baïonnettes, des lames, des lances : ce qui a été moissonné, ce sont des héros, tombés comme l'épi mûr.

No vulgar crop was theirs to reap,

No stinted harvest thin and cheap!

Heroes before each fatal sweep

Fell thick as ripened grain;

And ere the darkening of the day,

Piled high as autumn shocks, there lay

The ghastly harvest of the fray,

The corpses of the slain.

Qui prend la peine de regarder le paysage y décèle encore les sillons creusés par l'artillerie ou les limites du bivouac. Si l'herbe pousse, c'est que la terre a été enrichie de sang. Ce n'est qu'alors que le poème peut donner à voir, dans une série d'hypotyposes, l'horreur du combat, qui contraste avec le calme de la ville proche de Bruxelles. Scott, qui n'hésite pas à inclure des allégories, donne aussi droit de cité à des noms propres, non seulement «Bloody Waterloo », mais encore Napoléon puis Wellington, qui sort grandi du poème. La Bérézina ou le Pont de Lodi aussi, entre autres, sont mentionnés, dans ce texte qui présente les annales de deux décennies de combats sanglants.

Scott, dans sa fresque, est aussi l'un des seuls à évoquer le sort des veuves et des orphelins, avant de dresser son propre monument aux morts en citant les noms de Britanniques tombés au champ d'honneur : Picton, Ponsonby, De Lancey, Miller, Cameron et Gordon et d'indiquer que jamais on n'oubliera les braves victimes du combat :

Oft may the tear the green sod steep,

And sacred be the heroes' sleep,

Till time shall cease to run;

And ne'er beside their noble grave,

May Briton pass and fail to crave

A blessing on the fallen brave

Who fought with Wellington!

Le champ de Waterloo serait comme un visage à jamais marqué de cicatrices terribles, la mémoire du poète devient le magasin des images de la désolation. Il sait déjà qu'il a vu le lieu 
de l'une des grandes batailles de l'histoire, une bataille qui dans la violence de son choc en ferait oublier d'autres pourtant mémorables :

Yes-Agincourt may be forgot,

And Cressy be an unknown spot,

And Blenheim's name be new;

But still in story and in song,

For many an age remembered long,

Shall live the towers of Hougomont

And Field of Waterloo!

Le poète termine par une célébration de sa nation, reine des océans, victorieuse avec l'Europe. The Field of Waterloo $^{22}$ est un texte composé pour lever des fonds destinés à venir en aide aux veuves et aux orphelins des soldats tués lors des combats. Il devait avoir une conséquence annexe, qui n'était pas prévue, c'est de consacrer véritablement en lieu de mémoire le terrain de l'affrontement militaire et d'en faire un site de pèlerinage, plus marquant que les champs de bataille où se déroulèrent d'autres combats fameux. On peut remarquer que le guide vert de l'époque, The Traveller's Complete Guide to Belgium and Holland, publié par Charles Campbell en 1815, devait comprendre, à partir de 1817, dans une version augmentée, un passage intitulé $A$ Walk over the Field of Battle at Waterloo qui inclut des citations de différents tributs poétiques, entre autres des vers de Scott. Le poème fut rédigé plus rapidement que le volume en prose puisqu'il parut dès octobre 1815 (le recueil de lettres ne sortit qu'en janvier 1816). L'ouvrage se vendit bien avant tout, semble-t-il, grâce à la cause défendue : la presse fut assez critique - alors que Paul's Letters to his Kinsfolk fut salué par l'ensemble des médias. Il y eut trois éditions dans l'année après un premier tirage de 6000 exemplaires. Un anonyme eut le dernier mot sur la chute de l'œuvre :

On Waterloo's ensanguined plain

Full many a gallant man was slain,

But none, by sabre or by shot,

Fell half so flat as Walter Scott.

Le tourisme poétique est mis en évidence dans le choix de titre du poète lauréat, Robert Southey (1774-1843), qui se rendit sur les lieux au moment même où sortaient les vers de son jeune rival. Southey, qui est assez oublié de nos jours, jouissait d'une certaine célébrité. Il avait écrit une vingtaine d'années plus tôt un poème anti-militariste After Blenheim, et il avait, comme d'autres de sa génération, été enthousiasmé par les débuts de la Révolution. The Poet's Pilgrimage to Waterloo laisse entendre qu'il y a un devoir de célébration et de mémoire, lié peut-être aux fonctions officielles du lauréat dont la fonction est indiquée sur la page de titre. Le poème, précédé par un long préambule versifié et deux pages d'introduction en prose qui espèrent voir arriver un temps de paix et de floraison des arts, comprend 55 sixains. Waterloo est «the field of blood », à trois heures au Sud de Bruxelles, sauf pour un cavalier pressé qui peut rallier le site en un peu plus d'une heure. La campagne n'est pas belle, Waterloo est un petit village humble, obscur, devenu célèbre, un lieu rendu sacré par les cadavres des morts qu'on y a enterrés. C'est un champ d'honneur étranger, annexé en quelque sorte post mortem par les victimes :

\footnotetext{
${ }^{22}$ The Field of Waterloo; A Poem. By Walter Scott, Esq. Edinburgh; Printed by James Ballantyne and Co. For Archibald Constable and Co. Edinburgh; And Longman, Hurst, Rees, Orme, and Brown, and John Murray, London, 1815. - James Hogg a également écrit un poème sous le titre The Field of Waterloo. Il y fait allusion dans une lettre à Scott du 16 novembre 1815. Il l'a entrepris sans visiter le champ de bataille. Nous l'avons exclu de notre corpus.
} 
And here on marble tablets set on high,

In English lines by foreign workmen traced,

Are names familiar to an English eye;

Their brethren here the fit memorials placed,

Whose unadorned inscriptions briefly tell

Their gallant comrades' rank, and where they fell.

La noble simplicité de ces inscriptions provoque l'émotion. Le cimetière réunit un homme du cru, un Allemand venu pour combattre l'ennemi français, un Irlandais. L'Europe des nations serait fondée en quelque sorte dans le sang des victimes de cette bataille que Southey aussi décrit comme devant faire oublier tous les autres combats :

And even our glorious Blenheim to the field

Of Waterloo and Wellington must yield.

Le poète donne des indications géographiques, des toponymes, il évoque Hougomont, Papelot, le Mont Saint Jean ou encore La Haie Sainte ${ }^{23}$ et la Belle Alliance ${ }^{24}$ dont le nom acquiert une résonance nouvelle. Il montre la nature reprenant ses droits, effaçant les vestiges de la terrible guerre. Comme Byron, il unit dans ses vers les ennemis d'antan, les cavaliers et leurs chevaux ${ }^{25}$. Une terre matricielle les reprend comme elle paraît leur avoir donné naissance, sans bruit. Les traces des sabots restent, on trouve encore des reliques des combattants, une ceinture, des chapeaux, des pierres à fusil. Rappelons au passage qu'il a été beaucoup glosé sur le fait que les dents récupérées sur certains cadavres et transformées en prothèses ont conduit à la désignation des dentiers de l'époque par le nom de Waterloo teeth... Selon Southey, même l'air paraît parfois contaminé des restes de la mort, mais des pensées et des coquelicots fleurissent désormais sur le champ de bataille. Il se demande s'il faut tirer une quelconque consolation de ce cours de la nature qui fait mûrir les poires quelques mois après un tel choc.

Southey indique bien que la tâche du poète n'est pas celle de l'historien. Ils s'inscrivent dans des paradigmes différents ${ }^{26}$. Il rapporte des souvenirs de sa visite, d'avoir bavardé avec un jardinier qui était présent et livre son histoire - comme un avatar du témoin qui proposera, dans Les Misérables, de raconter, pour trois francs, «la chose de Waterloo ». Il rend une tache de sang à hauteur d'homme, sur un mur, emblématique de tout le sang versé. Pour lui, c'est Hougoumont, avec ses murs abîmés, mais aussi son jardin, qui devrait rester à jamais le mémorial de Waterloo :

LV.

Thy ruins as they fell should aye remain,...

What monument so fit for those below?

Thy garden through all ages should retain

\footnotetext{
${ }^{23}$ Behold the scene where Slaughter had full sway!/ A mile before us lieth Mount St. John,/ The hamlet which the Highlanders that day/ Preserved from spoil; yet as much farther on/ The single farm is placed, now known to fame,/ Which from the sacred hedge derives its name.

${ }^{24}$ Straight onward yet for one like distance more,/ And there the house of Belle Alliance stands,/ So named, I guess, by some in days of yore,/ In friendship, or in wedlock joining hands:/ Little did they who called it thus foresee/ The place that name should hold in history!

${ }^{25}$ Earth had received into her silent womb/ Her slaughtered creatures: horse and man they lay,/ And friend and foe, within the general tomb./ Equal had been their lot; one fatal day/ For all,.. one labour,... and one place of rest/ They found within their common parent's breast.

${ }^{26}$ XXXLI. This were the historian's, not the poet's part;/ Such task would ill the gentle Muse beseem,/ Who to the thoughtful mind and pious heart,/ Comes with her offering from this awful theme;/ Content if what she saw and gathered there/ She may in unambitious song declare.
} 
The form and fashion which it weareth now,

That future pilgrims here might all things see,

Such as they were at this great victory.

S'il termine ainsi sur le mot «victoire », contraint peut-être en partie par ses fonctions officielles, Southey n'écrit pas un poème de la célébration. Il rédige une sorte de guide en vers du champ de bataille. On peut imaginer que des touristes ont dû visiter les lieux le volume à la main. Southey en fait l'équivalent des grands sites de l'Antiquité, imaginant un sta viator, une stèle sur laquelle une inscription, porteuse prétendument de la parole des Ecossais qui ont péri, pourrait enjoindre au lecteur de retourner dire à leurs compatriotes combien ils ont été valeureux ${ }^{27}$. Parmi les combattants, Southey mentionne le prince d'Orange ( « young Nassau») dont il célèbre le courage, Picton, tombé au champ d'honneur, mais aussi des Français morts en nombre et un soldat inconnu, métonymie de toutes les victimes.

Another grave had yielded up its dead,

From whence to bear his son a father came,

That he might lay him where his own grey head

Ere long must needs be laid. That soldier's name

Was not remembered there, yet may the verse

Present this reverent tribute to his herse.

D'autres grands poètes de langue anglaise firent aussi le déplacement. En février 1816, ce fut le tour de Wordsworth (1770-1850), qui connaissait sans doute déjà les vers de Scott. De sa propre visite au champ de Waterloo, Wordsworth rapporta trois poèmes brefs. Nous sommes loin de la valeur documentaire présente chez Scott et, surtout, chez Southey. Wordsworth réclame pour le poète le rôle de chantre de la victoire. Il imagine une déesse, aux couleurs de l'arc-en-ciel, flottant au-dessus des lieux, avec d'innombrables couronnes pour les fronts des soldats comme pour leurs tombes. Elle disparaît et laisse un horizon vide. Le cœur lourd, l'on ne peut que penser au coût humain de la bataille :

While glory seemed betrayal, while patriot zeal

Sank in our hearts, we felt as Men should feel

With such vast hoards of hidden carnage near,

And horror breathing from the silent ground.

Le champ est vide. Face à une réalité absurde, Wordsworth réaffirme la primauté du vers comme monument aux morts, dans un exegi monumentum tragique :

To you who fell, and you who slaughter spared

To guard the fallen, and consummate the event,

Your Country rears this sacred Monument.

Avec la visite du champ de bataille, sorte de ground zero du monde contemporain pour les hommes et femmes du premier $\mathrm{XIX}^{\mathrm{e}}$ siècle, les écrivains sont confrontés à la nécessité de rendre compte d'une expérience double : le choc du combat d'un côté ; de l'autre, le vide qui en résulte, et qui fait qu'au fond, à Waterloo - avant la construction du lion, il n'y a pour ainsi dire rien à voir.

\footnotetext{
${ }^{27}$ XXVII. Here was it that the Highlanders withstood/ The tide of hostile power, received its weight/ With resolute strength, and stemmed and turned the flood;/ And fitly here, as in that Grecian straight,/ The funeral stone might say, Go traveller, tell/ Scotland, that in our duty here we fell.
} 
La poésie anglaise la plus célèbre sur Waterloo est vraisemblablement un passage d'un poème plus long, publié par étapes, Childe Harold's Pilgrimage de Byron, dont le héros est une figure romantique de jeune homme qui traîne son ennui dans toute l'Europe. Il partage certains traits de caractère avec son créateur, lequel, tout comme Scott et d'autres, a visité le champ de bataille. Byron s'y rend en effet en touriste sur la route de la Suisse en mai 1816. Pour lui, avec l'écroulement de l'Empire et la restauration monarchique, c'est un retour en arrière qui s'esquisse, une perte d'illusions.

Dans son poème héroï-comique Don Juan (1818-24), Byron s'adresse à Wellington pour lui dire qu'il serait heureux de savoir qui, à part le duc et les siens, peut se réjouir de ce qu'il est advenu à Waterloo. Le combat marque l'imaginaire du poète qui y revient dans plusieurs textes. L'allusion ironique est notamment précédée par un développement plus long dans une œuvre antérieure que nous avons déjà mentionnée, Childe Harold's Pilgrimage (181218). Le passage est souvent extrait de l'ensemble, traité comme un morceau à part que l'on inclut dans les anthologies. Il démarre par une évocation de la veille du combat, à l'occasion d'un bal lors duquel tout semble parfait. C'est le bruit du canon qui fait irruption dans les salons feutrés de la duchesse de Richmond et porte à Brunswick l'annonce de sa mort, au duc de Wellington et à son entourage l'annonce de la fin d'un monde. Byron dira ensuite le sang versé et l'horreur de la bataille. Vocabulaire et rythme montrent ce passage de l'équilibre à l'excitation de la bataille, de la mesure à la violence, de la joie des mondanités - à l'ouverture on entend «A sound of revelry by night » - au chaos et au bruit de la bataille : «Arm ! Arm ! And out - it is the cannon's opening roar!»Si Byron voyait la restauration monarchique comme mettant fin à la parenthèse de liberté, il propose aussi des raccourcis saisissants entre les délices mondaines et la violence meurtrière des combats. Ceux qui partent verront leur élan coupé court et seront, avant le soir, fauchés comme les blés. Il n'y aura plus ni victorieux, ni vaincu, ni cheval, ni cavalier, ni ami, ni ennemi. Tout se confond dans une terre rougie par le sang.

\author{
Last night beheld them full of lusty life, \\ Last eve in Beauty's circle proudly gay, \\ The midnight brought the signal-sound of strife, \\ The morn the marshalling in arms, - the day \\ Battle's magnificently-stern array! \\ The thunder-clouds close o'er it, which when rent \\ The earth is covered thick with other clay, \\ Which her own clay shall cover, heaped and pent, \\ Rider and horse, - friend, foe, - in one red burial blent!
}

Le souvenir personnel perce dans certains vers. Byron qui peine à voir Waterloo comme une victoire pleure son cousin Frederick Howard qui n'en revint point. Individu nommé, loué dans un passage élégiaque, il devient aussi une sorte de métonymie, un soldat connu, plutôt qu'inconnu, mais dont l'exemple témoigne du manque ressenti par tous ceux dont un proche a péri.

Le texte a la particularité de démarrer dans un avant-Waterloo festif alors que les autres poésies sont essentiellement des évocations du champ de bataille. Il restitue l'atmosphère enfiévrée de départ, lorsque tout le monde est dans l'expectative, se doutant qu'il va se passer un événement mémorable, mais sans savoir quand, ni où, ni comment. Le coup de canon qu'entend Brunswick et qu'il interprète à juste titre comme une annonce de sa propre mort, est aussi un présage du désastre à venir. 
Pour Byron, Waterloo est un concentré de toute l'histoire ainsi qu'en témoigne la référence implicite à Golgotha dans la strophe XVIII de Childe Harold. Comme d'autres jeunes gens de son époque, Byron, tout Britannique qu'il était, a admiré Napoléon. Il voit donc sa chute comme un épisode sanglant dans l'histoire, mais pas forcément comme une victoire.

And Harold stands upon this place of skulls,

The grave of France, the deadly Waterloo!

How in an hour the Power which gave annuls

Its gifts, transferring fame as fleeting too! -

In "pride of place" here last the Eagle flew,

Then tore with bloody talon the rent plain,

Pierced by the shaft of banded nations through;

Ambition's life and labours all were vain -

He wears the shattered links of the World's broken chain.

Dans l'inversion de la joie initiale à la confusion de la bataille, il y a l'image d'un monde qui s'écroule. L'ordre devient chaos.

And there was mounting in hot haste; the steed,

The mustering squadron, and the clattering car,

Went pouring forward with impetuous speed,

And swiftly forming in the ranks of war;

And the deep thunder, peal on peal afar;

And near, the beat of the alarming drum

Roused up the soldier ere the morning star;

While thronged the citizens with terror dumb,

Or whispering, with white lips - "The foe! they come! they come!"

L’ennemi est là. Le combat aura lieu.

L'on pourrait s'attendre à des poèmes de célébration dans la grande tradition des éloges versifiés à l'occasion de triomphes militaires, mais les faits sont tout autres dans les œuvres dont on se souvient ${ }^{28}$. En effet, la poésie de Waterloo frappe à bien des égards par son ambiguïté.

On peut observer que s'il y a eu des visites à des grands hommes vivants comme Voltaire ou sur des lieux immortalisés dans des textes pour leur beauté, comme les Alpes suisses ou les rives du Léman, ce tourisme militaire est particulier ${ }^{29}$. Certains vont rechercher l'horreur. On peut songer au peintre Turner, directement influencé par les vers de Byron, qui rapporte de son propre voyage des esquisses des lieux, entre autres de la ferme d'Hougomont, mais aussi l'inspiration d'un tableau exposé pour la première fois en 1818 et qui montre un champ de cadavres parmi lesquels des femmes endeuillées cherchent les leurs ${ }^{30}$.

\footnotetext{
${ }^{28}$ Il y a en effet quelques poèmes « nationalistes » dans la presse etc.

${ }^{29}$ On peut se demander si la mise en valeur de différents champs de bataille, comme Bannockburn, près de Stirling, etc., sans parler de Pearl Harbor à Hawaii, ne suit pas une sorte de mode lancée par le tourisme à Waterloo.

${ }^{30}$ Signalé par Philip Shaw, Waterloo and the Romantic Imagination, 2002. - Turner ne répond pas à l'appel lancé par la British Institution pour le meilleur tableau de la bataille dès 1816, et que devait remporter, pour une œuvre perdue, James Ward.
} 
J'aurais pu évoquer d'autres vers anglais, comme «The Battle of Waterloo ${ }^{31}$ » du très oublié William Thomas Fitzgerald qui avait déjà publié un recueil contre Napoléon. Dans son texte sur Waterloo, il est question, avec force majuscules, du COUPABLE, appelé au TRIBUNAL de l'Europe, du CORSE DETESTE enfin vaincu, de l'extraordinaire WELLINGTON. J'ai préféré m'attarder sur d'autres textes dans lesquels les sentiments sont plus nuancés. Un des éléments frappants est en effet que, de part et d'autre de la Manche, les grands poètes, Delavigne, Scott, Wordsworth, Byron, refusent tous la forfanterie, la glorification de la guerre. Tous sont sensibles au coût humain des combats. Tous espèrent un avenir meilleur et reconnaissent les qualités de leurs adversaires. Si Napoléon est tour à tour exalté ou loué, les soldats sont vus dans leur humanité face à un événement qui déshumanise. Enracinés, pour les poètes de langue anglaise, dans l'expérience d'un pèlerinage littéraire sur les lieux de la bataille, les vers, de part et d'autre de la Manche, aspirent, à leur façon, à se faire monuments mais, le plus souvent, dans l'esprit du Poème sur Fontenoy de Voltaire, par l'exaltation du soldat, plus que du général, dans la volonté programmatique, également, de signaler une fin de l'horreur, d'appeler à l'apaisement des passions.

Que reste-t-il de Waterloo dans la poésie ? Pour les francophones, c'est « L'Expiation» de Victor Hugo, assez célèbre pour que Goscinny et Uderzo y fassent référence dans Asterix chez les Belges par allusion gastronomique interposée. La blague de potache cultivé (le guerrier belge regarde sans enthousiasme le repas qui lui est servi en soupirant «Waterzooie! Waterzooie ! Waterzooie ! morne plat ! ») est divertissante et repose, pour qu'on en saisisse la pleine saveur, à la fois sur l'identification d'un des plats phares de la cuisine belge, et sur une reconnaissance de la citation détournée. C'est une réaction tardive, que celle d'Hugo dans «L'Expiation ». Du côté des textes anglais, la référence poétique immédiate à Waterloo pour tout lecteur cultivé est, à mon sens, à chercher chez Byron. Là encore je prendrai comme témoignage et confirmation les aventures d'Astérix, mais dans la traduction anglaise. Si la scène du dîner d'avant la bataille n'entraîne pas de légende poétique, mais plutôt une allusion à une expression populaire avec un jeu anachronique divertissant selon lequel César va être vaincu will meet his Waterloo - il y a ailleurs dans l'album trois citations du passage qui concerne Waterloo du poème de Byron, Childe Harold's Pilgrimage, dont le célèbre incipit, de ce qu'on appelle The Eve of Waterloo ${ }^{32}$ transformé en commentaire sur un repas festif qui doit beaucoup, en termes artistiques, à Breughel. J'en profite pour saluer le remarquable travail sur les transferts culturels des traducteurs de la bande dessinée, Anthea Bell et Derek Hockridge.

Les poètes l'ont montré : Waterloo a le statut d'événement fondateur de l'Europe moderne, mais permet à chacun d'y réagir avec sa sensibilité. Par leurs écrits, ils réclament de toute évidence pour le poète un rôle d'historiographe. Ils aident à donner une valeur mythique à ce qu'ils voient comme un choc des titans, comme l'écroulement d'un monde, comme une bataille dans laquelle chacun est, jusqu'à un certain point, perdant.

\footnotetext{
${ }^{31}$ The Battle of Waterloo "Wm. Thos. Fitzgerald, Esq." [William Thomas Fitzgerald], The New Monthly Magazine, IV (August 1815), p. 34-35; The Gentleman's Magazine, LXXXV, ii (1815), p. 62; The European Magazine, LXVIII (1815), p. 55-56.

${ }^{32}$ Nearer, clearer, deadlier than before... renvoie à Childe Harold's Pilgrimage, de même que There was a sound of revelry by night ou encore On with the dance. Let joy be unconfined.
} 